\title{
SIBYLS - a SAXS and Protein Crystallography Beamline at the ALS
}

\author{
C.Trame*, A.A.MacDowell*, R.S.Celestre*, H.A.Padmore*, D.Cambie*, \\ E.E.Domning*, R.M.Duarte*, N.Kelez*, D.W.Plate*, J.M.Holton*, K.Frankel*, \\ S.Tsutakawa*, H.Tsuruta ${ }^{\#}$, J.A.Tainer* ${ }^{\dagger}$, P.K.Cooper*. \\ * Lawrence Berkeley National Lab., 1 Cyclotron Road, Berkeley, CA 94720, USA \\ \# Stanford Synchrotron Radiation Lab., 2575 Sand Hill Road, Menlo Park, California, 94025, USA \\ ${ }^{\dagger}$ Scripps Research Institute, 10550 North Torrey Pines Road, La Jolla, CA 92037
}

\begin{abstract}
The new Structurally Integrated BiologY for Life Sciences (SIBYLS) beamline at the Advanced Light Source will be dedicated to Macromolecular Crystallography (PX) and Small Angle X-ray Scattering (SAXS). SAXS will provide structural information of macromolecules in solutions and will complement high resolution PX studies on the same systems but in a crystalline state. The x-ray source is one of the 5 Tesla superbend dipoles recently installed at the ALS that allows for a hard x-ray program to be developed on the relatively low energy Advanced Light Source (ALS) ring $(1.9 \mathrm{GeV})$. The beamline is equipped with fast interchangeable monochromator elements, consisting of either a pair of single $\mathrm{Si}(111)$ crystals for crystallography, or a pair of multilayers for the $\mathrm{SAXS}$ mode data collection $(\mathrm{E} / \Delta \mathrm{E} \sim 1 / 110)$. Flux rates with $\mathrm{Si}(111)$ crystals for $\mathrm{PX}$ are measured as $2 \times 10^{11} \mathrm{hv} / \mathrm{sec}$ through a $100 \mu \mathrm{m}$ pinhole at $12.4 \mathrm{KeV}$. For SAXS the flux is up to $3 \times 10^{13}$ photons/sec at $10 \mathrm{KeV}$ with all apertures open when using the multilayer monochromator elements. The performance characteristics of this unique beamline will be described.
\end{abstract}

\section{INTRODUCTION}

At synchrotrons around the world, Protein Crystallography beamlines are being built at a rapid pace as it has been realized that this technique can quickly elucidate protein structures, which can provide one of the foundation blocks for the understanding of life. At the Advanced Light Source (ALS) the latest and the seventh Protein Crystallography beamline has recently come one line. This paper describes the technical details. The beamline is known as the SIBYLS beamline (Structurally-Integrated BiologY for Life Sciences), the aim being to bring many of the synchrotron based techniques together on on beamline. To this end, the beamline is equipped with a standard Protein Crystallography (PX) setup and has the additional capability of being able to carry out Small Angle Scattering (SAXS) with time resolved scattering studies on solutions. To achieve this, the beamline has a SAXS and a PX end station, with additional spectroscopic equipment for absorption and fluorescence measurements in the visible and ultra violet. Ease of interchange ability between PX and SAXS mode has been an important requirement in this design.

\section{SOURCE, BEAMLINE AND END-STATION DESCRIPTION}

The beamline is a development on the previous three successful PX beamlines [1] deployed at the ALS with the addition of having a monochromator with both $\mathrm{Si}(111)$ and multilayer monochromator elements and an end station capable of carrying out PX and static and time resolved SAXS.

The x-ray source is one of the three 5 Tesla superbend dipoles recently installed around the ALS. These allow for a hard $\mathrm{x}$-ray program to be developed on a relatively low energy $(1.9 \mathrm{GeV})$ ring. The ALS storage ring lattice is 12 fold symmetric with the basic magnet lattice of the triple bend achromat type [2]. Three of the thirty six 1.27 Tesla warm, 10 degree, bending magnets (critical energy $=3 \mathrm{KeV}$ ) were replaced by 5 Tesla, superconducting, 10 degree, bending magnets (critical energy $=12 \mathrm{KeV}$ ). $[3,4]$. This was done by modifying 3 of the 12 sectors. The retrofitting of the storage ring with superconducting dipoles was very challenging and the first ever operation of this type carried out on an operating synchrotron radiation source. The Superbends would be an essential part of the ring lattice and problems associated with them would affect all users of the ring. Extensive beam dynamic studies were carried out beforehand, and all systems were modelled and tested prior to installation. Commissioning proved to be straightforward and after more than 18 months of use, the Superbends appear transparent to the rest of the users on the ring. 
The basic beamline layout was the same employed on the previous PX superbend beamlines [1]. The beamline design adopted is shown schematically in Fig.1. It consists of the source, a vertically deflecting plane parabolic collimating mirror (grazing angle $=4.5 \mathrm{mrad}$, acceptance $=1.5 \times 0.5 \mathrm{mrad}$ (horizontal (h) $\mathrm{x}$ vertical (v)) that provides parallel radiation for the monochromator (two flat crystals or two flat multilayers), followed by a toroidal mirror that focuses the light into the hutch on either the SAXS detector for regular SAXS work, the PX sample for regular PX or the PX detector. The later mode of operation allows for the analysis of large unit cells to high resolution. We positioned the SAXS station upstream and in series with the PX station inside of a large hutch. The SAXS station can be entirely retracted to allow for operation of the PX station (see later). The change in focus location is achieved by slight change of the M2 tilt angle. Previously [1] we found that when the toroidal mirror is used with parallel illumination in the vertical and a 2:1 demagnification in the horizontal, then the main aberrations cancel out and a high quality image results. This has been determined to be due to the vanishing of the astigmatic coma for the 2:1 horizontal demagnification case [5]. Using an electron source size of $230 \times 23 \mu \mathrm{m}$ fwhm (h x v), the focused spot size is ray traced to be $150 \times 63 \mu \mathrm{m}$ fwhm (h x v) with a beamline acceptance of $1.5 \times 0.5 \mathrm{mrad}(\mathrm{h} \mathrm{x}$ v). True diffraction limited imaging with horizontal and vertical demagnifications of 2.0 and 0.62 respectively should produce an image of size $115 \times 37 \mu \mathrm{m}$ fwhm (h x v), so this optical layout does result in some brightness loss, however as the sample phase space is under filled in the vertical, flux loss is not huge and is more than offset by the operation considerations associated with the mechanical robustness of the design. We have chosen the PX sample location to be at this 2:1 demagnification location. The other 2 focus locations are slightly upstream or downstream of the optimum focus and thus focus degradation is very slight.

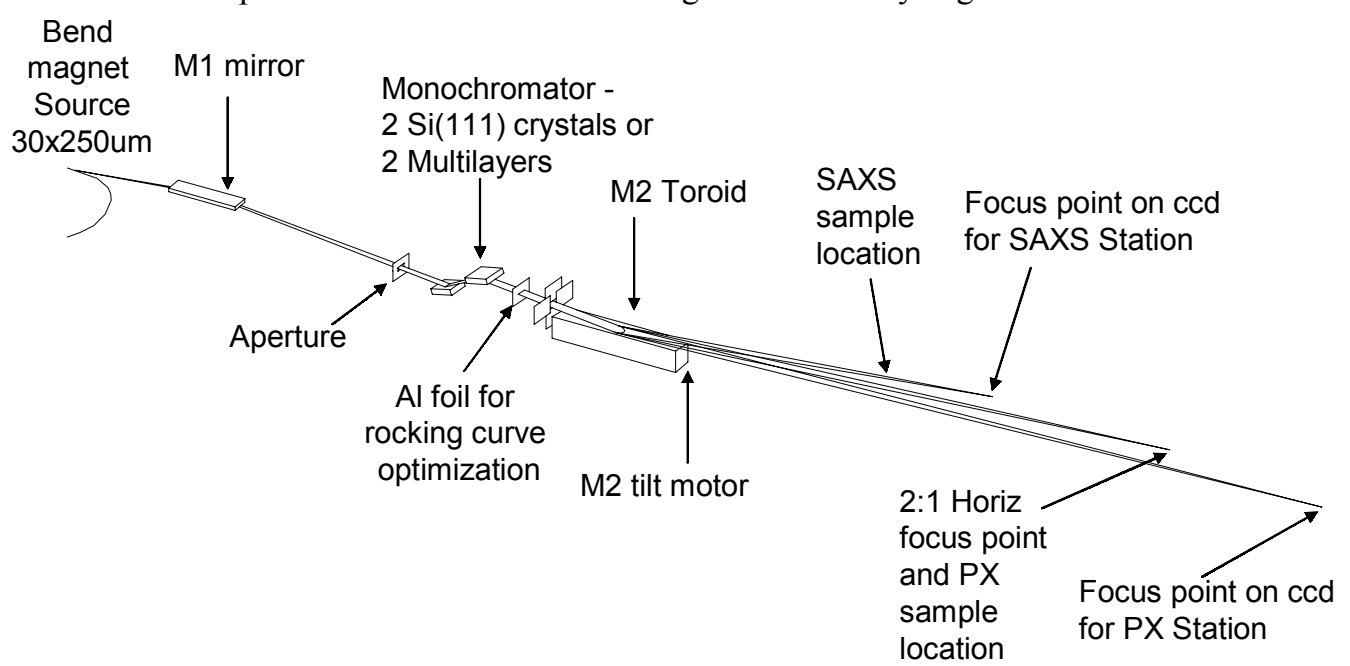

FIGURE 1. Schematic layout of the SIBYLS Beamline with a Superbend dipole magnet source. The beamline acceptance is $1.5 \times 0.5 \mathrm{mrad}$ (horizontal $\mathrm{x}$ vertical). The distances of the M1 plane paraboloid mirror, monochromator, M2 toroid from the source are $6.5 \mathrm{~m}, 15.5 \mathrm{~m}, 20 \mathrm{~m}$ respectively. The source distance to SAXS sample, SAXS detector, PX sample, and PX detector (when furthest from sample) are $27.15 \mathrm{~m}, 28.85 \mathrm{~m}, 30 \mathrm{~m}$ and $31.6 \mathrm{~m}$.

The optics used have the following descriptions. M1 is a flat internally water cooled electroless Nickel plated Invar mirror. It is held in a mechanical bender that allows it to be bent to the required parabolic shape. The M2 mirror is an uncooled silicon cylinder mirror bent to a toroid by means of a mechanical bender. Both mirrors are coated with $8 \mathrm{~nm}$ of Rhodium over $25 \mathrm{~nm}$ of Platinum and operated at $4.5 \mathrm{mrad}$ grazing angle. This bilayer coating allows for suppression of the Platinum $\mathrm{L}$ edges in the (11-14) KeV range whist extending the mirror cut off $\sim 2.5 \mathrm{KeV}$ beyond what one would obtain from a single Rhodium coated mirror [1]. The first crystal is an internally water cooled flat $\mathrm{Si}(111)$ crystal, the first multilayer is a side cooled flat with 150 layer pairs of $\mathrm{Mo} / \mathrm{B}_{4} \mathrm{C}, \mathrm{d}$ spacing $=$ $2.4 \mathrm{~nm}$. The second crystal and multilayer are simple uncooled flat elements.

The monochromator is a standard Kohzu type APM monochromator [6] with custom in-house additions for the crystals and multilayer optics. Fig.2 shows the schematic layout. The basic monochromator rotates all optics about the central axis that runs along the surface of crystal 1 . This rotation is the main Theta drive that determines the monochromator angle and energy. The first crystal is fixed to this main rotation platform, whilst the second crystal is mounted off a stack of 4 stages that in turn is supported from the main Theta rotation platform. The 4 stages that the second crystal rides on can be adjusted to maintain the constant beam height offset required for the beamline optics. The first multilayer is fixed to the first crystal support structure and as shown in Fig.2 is mounted upstream of the first crystal. The second multilayer is mounted on the stack of 4 stages with the second $\operatorname{Si}(111)$ crystal. By 
rotating the main Theta drive to the low grazing angle required for the multilayer, its location is selected such that its off axis rotation intercepts the beam before the $\mathrm{Si}(111)$ crystal and directs the x-rays to the second multilayer. Of course the off axis location of the first multilayer means that the $\mathrm{x}$-rays will walk off the surface, but by suitable choice of mirror length $(176 \mathrm{~mm})$ and $\mathrm{d}$ spacing $(2.4 \mathrm{~nm})$ it is possible to get a useful energy range of $\sim 7-13 \mathrm{KeV}$ before the light has walked too far off the mirrors.

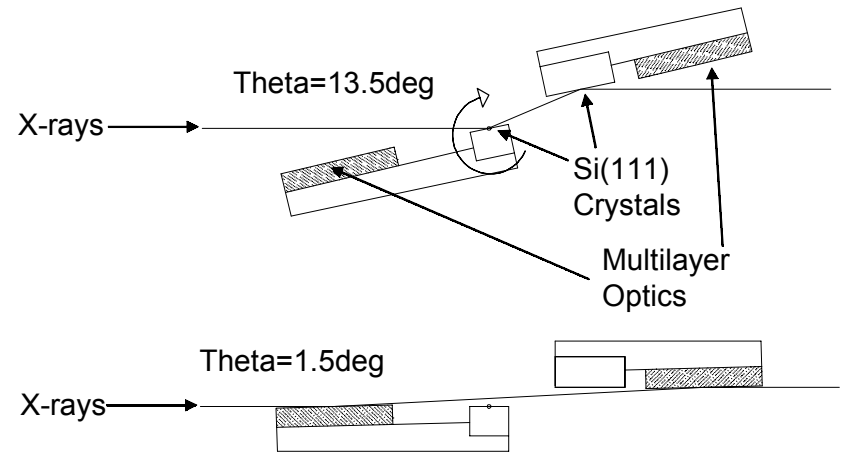

FIGURE 2. Schematic layout of the monochromator elements within the Kohzu monochromator.

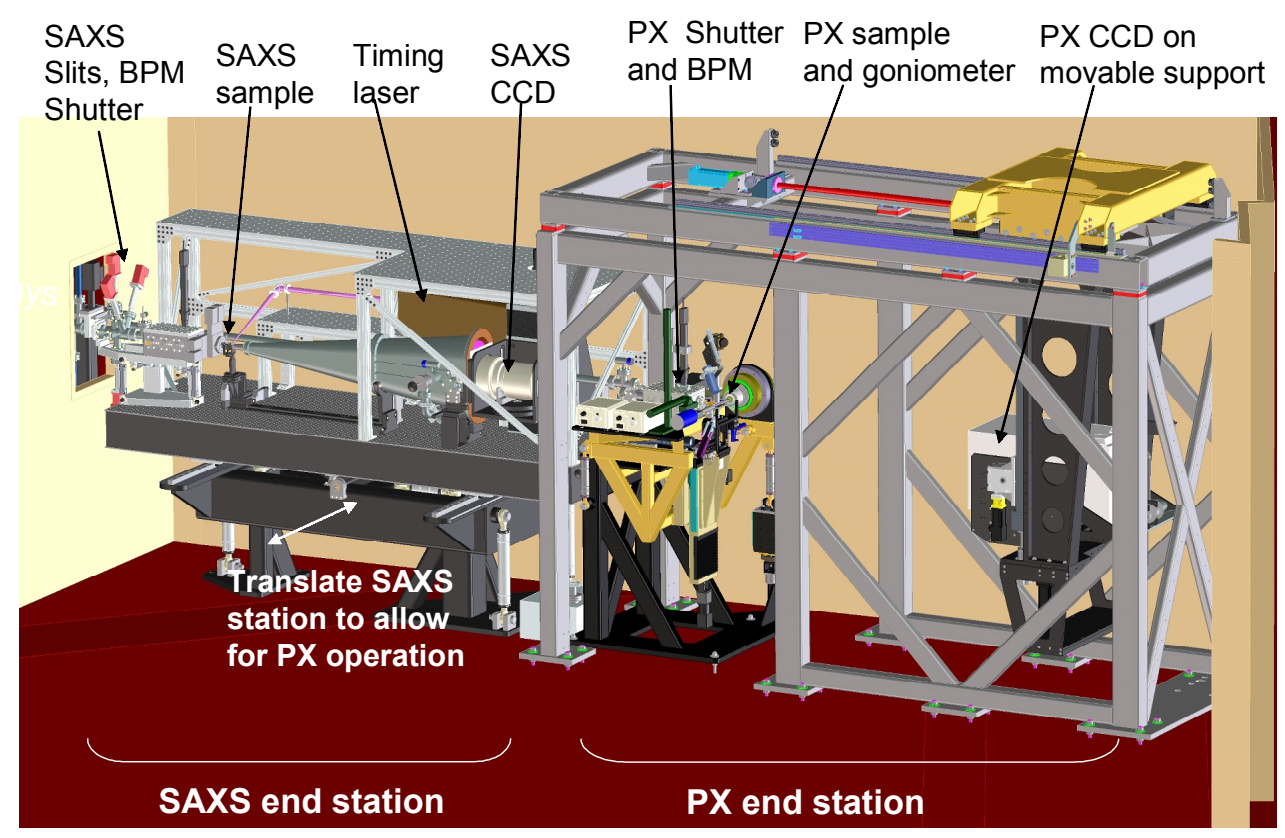

FIGURE 3. Engineering model of the SAXS and PX end stations. X-rays enter the hutch from the left. (BPM - beam position monitor).

The engineering models of the SAXS and PX end stations are shown in Fig.3. The SAXS and PX end stations are in series with the SAXS station located upstream. The beamline proper ends to the left of Fig. 3 with a Beryllium window and the hutch safety shutter. An air gap of $15 \mathrm{~mm}$ separates the beamline beryllium window from the SAXS beryllium window where the X-rays pass into a Helium filled section containing adjustable slits, beam position monitoring and a ccd shutter. The beam exits another beryllium window just before the sample. Following the sample is a $1.7 \mathrm{~m}$ evacuated flight tube before the Mar $165 \mathrm{CCD}$ camera. The camera is mounted on a vertical translation stage and allows for collection of data to high q $\left(0.38 \AA^{-1}\right)$. A timing laser system coupled to fiber optics will offer the ability to carry out kinetic studies and also spectroscopic investigations. The Mar CCD can be operated in frame transfer mode such that a time resolution of $\sim 50 \mathrm{~ms}$ can be achieved. The SAXS system is all mounted on a table that can be translated $30 \mathrm{~cm}$ to the side. The SAXS station is thus replaced by a long evacuated tube that allows $\mathrm{x}$-rays to the PX end station. Ease of transition between SAXS mode and PX mode is assured. The X-rays emerge from the long evacuated tube via a beryllium window before passing through $15 \mathrm{~mm}$ of air followed by a helium filled box containing beam position monitoring, ccd shutter and the final beryllium window. The beam then passes 
through a $100 \mu \mathrm{m}$ pinhole the sample and then a $300 \mathrm{um}$ back stop mounted with a Styrofoam support structure. The latter is invisible to x-rays, does not cast any shadow on the diffraction pattern and provides a good visible light scattering medium for back illumination when viewed along the $\mathrm{x}$-ray axis using the similar 2 zoom optical arrangement used before [1]. The diffraction pattern is collected by an ADSC Q315 ccd that can be located 0.12 $1.6 \mathrm{~m}$ from the sample with a 2-Theta Bragg range of -5 to 45 degrees.

Figure 4 shows the calculated and measured flux for the beamline. The second curve from the top is the multilayer case with no pinhole and represents typical fluxes that are available for SAXS experiments. Energy resolution was measured as $\mathrm{E} / \Delta \mathrm{E}=110$ at $8979 \mathrm{eV}$. These flux rates will be adequate for time resolved SAXS. The lowest curve is the $\mathrm{Si}(111)$ case when focused through a 100um pinhole just before the sample and represents the setup used for standard PX. Flux rates will allow exposure times of 1-10 secs dependant on sample quality. Focused spot size was measured as $165 \times 130 \mu \mathrm{m}$ fwhm (h X v), which is close to the modeled value in the horizontal, and a little larger in the vertical - most likely due to mirror slope errors.

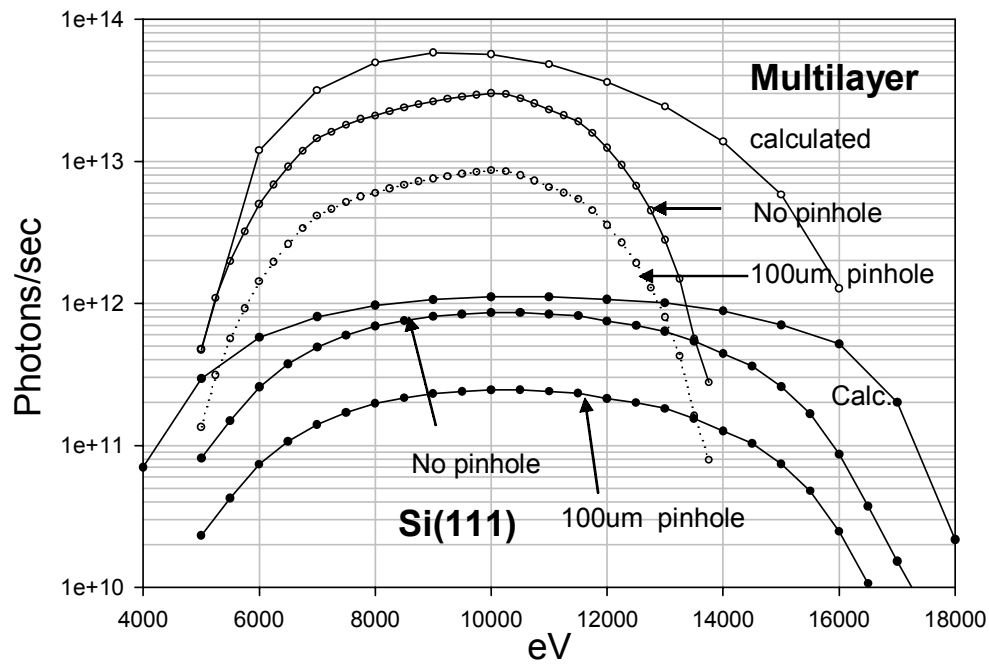

FIGURE 4. Plot of calculated and measured flux for the multilayer and Si(111) monochromator pairs at the PX sample position. The upper plot is the total calculated flux from the multilayer pair. The two plots slightly lower are the cases for the measured total flux at the sample location and the measured flux through a $100 \mu \mathrm{m}$ diameter pinhole. The lower 3 curves are similar plots for the $\mathrm{Si}(111)$ crystal pair. Flux rates are for $400 \mathrm{~mA}$ in the ALS and full acceptance of the beamline resulting in a convergence onto the sample of $3.0 \mathrm{mrad}(\mathrm{h}) \times 0.31 \mathrm{mrad}(\mathrm{v})$.

\section{ACKNOWLEDGMENTS}

The Advanced Light Source is supported by the Director, Office of Science, Office of Basic Energy Sciences, Materials Sciences Division, of the U.S. Department of Energy under Contract No. DE-AC03-76SF00098 at Lawrence Berkeley National Laboratory and by the National Institute of Health via grant CA92584-04.

\section{REFERENCES}

1. MacDowell, A.A., Celestre, R.S., Howells, M.R., Krupnick, J., Padmore, H.A., Cambie, D., Domning, D.E, Duarte, R.M., Kelez, N., Plate, D.W., Cork, C.W., Earnest, T.N., Dickert, J., Meigs, G., Ralston, C., Holton, J.M., Alber, T., Berger, S. and Agard, D.A. Submitted to J.Sync. Rad.

2. 1-2 GeV Synchrotron Radiation Source - Conceptual Design Report, Lawrence Berkeley National Laboratory Report PUB5172. July (1986)

3. Robin, D., Benjegerdes, R., Biocca, A., Bish, P., Brown, W., Calais, D., Chin, M., Corradi, C., Coulomb, D., De Vries, J., DeMarco, R., Fahmie, M., Geyer, A., Harkins, J., Henderson, T., Hinkson, J., Hoyer, E., Hull, D., Jacobson, S., Krupnick, J., Marks, S., McDonald, J., Mollinari, P., Mueller, R., Nadolski, L., Nishimura, K., Ottens, F., Paterson, J.A., Pipersky, P., Ritchie, A., Rossi, S., Salvant, B., Schlueter, R., Schwartz, A., Spring, J., Steier, C., Taylor, C., Thur, W., Timossi, C., Wandesforde, J. and Zbasnik, J. Proceedings of the 2002 European Particle Accelerator Conf., Paris, France, p. 215 (2002)

4. Tamura, L. and Robinson, A. Synchrotron Radiation News, 15, 30-34 (2002)

5. Howells, M.R., MacDowell, A.A., McKinney, W.R. \& Padmore, H.A. To be submitted to J.Synch. Rad. (2003)

6. Kohzu Seiki Co., 2-27-37 Mishuku, Setagaya-Ku, Tokyo, 154-0005, Japan. 